\title{
CASK mutations are frequent in males and cause X-linked nystagmus and variable XLMR phenotypes
}

\author{
Anna Hackett ${ }^{\star,}$, Patrick S Tarpey ${ }^{2}$, Andrea Licata ${ }^{3}$, James Cox ${ }^{4}$, Annabel Whibley ${ }^{4}$, Jackie Boyle ${ }^{1}$, \\ Carolyn Rogers $^{1}$, John Grigg ${ }^{5}$, Michael Partington ${ }^{1}$, Roger E Stevenson ${ }^{3}$, John Tolmie ${ }^{6}$, John RW Yates $^{4}$, \\ Gillian Turner ${ }^{1}$, Meredith Wilson ${ }^{7}$, Andrew P Futreal' ${ }^{2}$, Mark Corbett ${ }^{8}$, Marie Shaw ${ }^{8}$, Jozef Gecz ${ }^{8,9}$, \\ F Lucy Raymond ${ }^{4}$, Michael R Stratton ${ }^{2,10}$, Charles E Schwartz ${ }^{3}$ and Fatima E Abidi ${ }^{3}$
}

\begin{abstract}
Mutations of the calcium/calmodulin-dependent serine protein kinase (CASK) gene have recently been associated with $\mathrm{X}$-linked mental retardation (XLMR) with microcephaly, optic atrophy and brainstem and cerebellar hypoplasia, as well as with an X-linked syndrome having some FG-like features. Our group has recently identified four male probands from 358 probable XLMR families with missense mutations (p.Y268H, p.P396S, p.D710G and p.W919R) in the CASK gene. Congenital nystagmus, a rare and striking feature, was present in two of these families. We screened a further 45 probands with either nystagmus or microcephaly and mental retardation (MR), and identified two further mutations, a missense mutation (p.Y728C) and a splice mutation (c.2521-2A $>$ T) in two small families with nystagmus and MR. Detailed clinical examinations of all six families, including an ophthalmological review in four families, were undertaken to further characterise the phenotype. We report on the clinical features of 24 individuals, mostly male, from six families with CASK mutations. The phenotype was variable, ranging from non-syndromic mild MR to severe MR associated with microcephaly and dysmorphic facial features. Carrier females were variably affected. Congenital nystagmus was found in members of four of the families. Our findings reinforce the CASK gene as a relatively frequent cause of XLMR in females and males. We further define the phenotypic spectrum and demonstrate that affected males with missense mutations or in-frame deletions in CASK are frequently associated with congenital nystagmus and XLMR, a striking feature not previously reported.
\end{abstract}

European Journal of Human Genetics (2010) 18, 544-552; doi:10.1038/ejhg.2009.220; published online 23 December 2009

Keywords: CASK gene; XLMR; intellectual disability; congenital nystagmus

Over the past 10 years, the calcium/calmodulin-dependent serine protein kinase $(C A S K)$ gene has been identified as a candidate gene for X-linked optic atrophy and X-linked mental retardation (XLMR). ${ }^{1-3}$ Najm et al ${ }^{4}$ reported heterozygous inactivating mutations of CASK as causing microcephaly, brainstem and cerebellar hypoplasia, and severe mental retardation (MR) in four females, and a partly penetrant splice mutation causing a severe cerebral malformation in one male who died at the age of 2 weeks. More recently, Piluso et al ${ }^{5}$ reported a missense mutation in CASK causing an X-linked syndrome with some features that overlap the FG phenotype. In an effort to identify additional genes associated with XLMR, our group identified four male probands from 358 probable XLMR families as having novel missense mutations in CASK. ${ }^{6}$ The clinical features were generally mild-to-moderate MR, but with congenital nystagmus present in two families.

The CASK gene, a member of the MAGUK protein family, maps to Xp11.4 and encodes a CASK, a multi-domain scaffolding protein of 926 amino acids ${ }^{1}$ (Ensembl ID: ENST00000378163). The CASK protein consists of an N-terminal CAM-kinase domain, two L27 domains, a PDZ domain, an $\mathrm{SH} 3$ domain and a C-terminal guanylate kinase domain. ${ }^{7}$ It is located at both the postsynaptic membrane of central nervous synapses and within the nuclei of neurons and is ubiquitously expressed with a significantly higher expression in the foetal brain. ${ }^{8} 9$ Different domains of the CASK protein interact with more than a dozen different proteins and may be involved in synaptic interaction, protein trafficking and regulation of gene expression during neural development. ${ }^{9-12}$

To determine whether nystagmus and/or microcephaly associated with MR were good clinical indicators of CASK mutations, we screened an additional 45 probands with MR plus either nystagmus or microcephaly. Two families with a clinical phenotype of XLMR and nystagmus were identified; one with a novel missense mutation, p.Y728C, and one with a splice site mutation, c.2521-2A $>$ T. In this study, we present the details of molecular and clinical findings of six families with CASK mutations.

\footnotetext{
${ }^{1}$ Genetics of Learning Disability Service, Hunter Genetics, Waratah, New South Wales, Australia; ${ }^{2}$ Wellcome Trust Sanger Institute, Cambridge, UK; ${ }^{3}$ JC Self Research Institute, Greenwood Genetic Center, Greenwood, SC, USA; ${ }^{4}$ Cambridge Institute of Medical Research, Cambridge, UK; ${ }^{5}$ Save Sight Institute, Sydney Eye Hospital Campus, University of Sydney, Sydney, New South Wales, Australia; ${ }^{6}$ Institute of Medical Genetics, Yorkhill Hospital, Glasgow, UK; ${ }^{7}$ Department of Clinical Genetics, Children's Hospital at Westmead, Westmead, New South Wales, Australia; ${ }^{8}$ SA Pathology, Women's and Children's Hospital and Department of Paediatrics, The University of Adelaide, Adelaide, South Australia, Australia; ${ }^{9}$ Department of Paediatrics, The University of Adelaide, Adelaide, South Australia, Australia; ${ }^{10}$ Cancer Research UK Genetic Epidemiology Unit, Strangeways Research Laboratory, Cambridge, UK

*Correspondence: Dr A Hackett, Genetics of Learning Disability Service, Hunter Genetics, PO Box 84, Waratah, Newcastle, New South Wales 2298, Australia. Tel: +612 498 53136; Fax: +612 498 53105; E-mail: anna.hackett@hnehealth.nsw.gov.au

Received 11 August 2009; revised 22 October 2009; accepted 28 October 2009; published online 23 December 2009
} 


\section{METHODS}

\section{Patients}

As a part of the International Genetics of Learning Disability group, DNA from 208 unrelated male probands underwent bidirectional DNA sequencing of the coding exons of 720 Vega-annotated genes on the $\mathrm{X}$ chromosome, and a further 150 unrelated probands with XLMR had DNA sequence analysis performed on the CASK gene, as previously described. ${ }^{6}$ Sequencing of the CASK gene was performed in an additional 45 individuals: 25 ( 1 female, 24 male) with clinical findings of MR and nystagmus; 9 ( 1 female, 8 male) with MR, microcephaly and nystagmus; and 11 ( 2 female, 9 male) with MR, microcephaly and other eye disorders. Informed consent was obtained from all patients, relatives and control individuals for participating in this study.

\section{$\mathrm{X}$-inactivation studies}

$\mathrm{X}$-chromosome inactivation (XCI) was determined by methylation analysis of the androgen receptor polymorphic CAG repeat and/or the FMR1 gene variable CGG repeat. ${ }^{13,14}$

\section{cDNA preparation and RT-PCR}

Reverse transcriptase PCR (RT-PCR) was carried out using standard techniques as previously described. ${ }^{15,16}$

\section{RESULTS}

Sequencing of X-chromosome Vega-annotated genes

Two missense variants in CASK were identified from the cohort of 208 unrelated male probands. ${ }^{6}$

Family 74. Sequencing of the CASK gene revealed an A to G change at position 2129 (c.2129A $>\mathrm{G}$ ) within exon 22, which results in the substitution of glycine for aspartic acid at position 710 (p.D710G) and creates a new $5^{\prime}$ donor splice site that has an information content of 8.88 versus $0.70 .{ }^{17}$ RT-PCR analysis of cDNA prepared from a lymphoblastoid cell line from the proband indicated that the mutation resulted in aberrant splicing of exon 22, resulting in the removal of 27 nucleotides at the $3^{\prime}$-end of exon 22 (Figure 1). The resultant protein has an in-frame deletion of nine amino acids (p.710_718 Del DKYLAKHNA) at the C-terminal end of the 'Hook Motif' (the protein 4.1 binding motif) ${ }^{9}$ (Figure 2). The variant segregates with $M R$ and nystagmus in affected males and in the obligate female carrier, III-2 (Figure 3). Individual III-6 with mild MR and no nystagmus did not have the variant.

Family 16. Sequencing of the CASK gene revealed a $\mathrm{T}$ to $\mathrm{C}$ change at position 802 (c.802T $>$ C) in exon 8 , resulting in the substitution of histidine for tyrosine at position 268 (p.Y268H), located in the CaMKlike domain (Figure 2). The variant segregated with the MR phenotype in the family (Figure 3). Bioinformatic analysis did not indicate this variant as being pathogenic (Supplementary Table 1), although the tyrosine at position 268 is highly conserved (Figure 4 ).

\section{Sequencing of the CASK gene}

Two missense variants were identified in an additional 150 probands with XLMR, sequenced for mutations in the coding exons of the CASK gene. ${ }^{6}$

Family 123. A $\mathrm{T}$ to $\mathrm{C}$ change in exon 27 at position 2756 (c.2756T $>$ C) was identified, resulting in the substitution of arginine for tryptophan at position 919 (p.W919R) at the C-terminal end of the CASK protein (Figure 2). The variant segregated in the three affected males with MR and nystagmus and in their mother (Figure 3). Polyphen and SIFT analysis of this variant predicts it to be deleterious to the function of the protein (Supplementary Table 1). The tryptophan at position 919 is highly conserved across different species (Figure 4).

K8919 (family 245). A C to $\mathrm{T}$ change in exon 13 at position 1186 (c.1186C > T) was identified, resulting in a proline to serine substitution at position 396 (p.P396S), located in the first L27 domain, also known as L27.1 (Figure 2). The variant was present in the
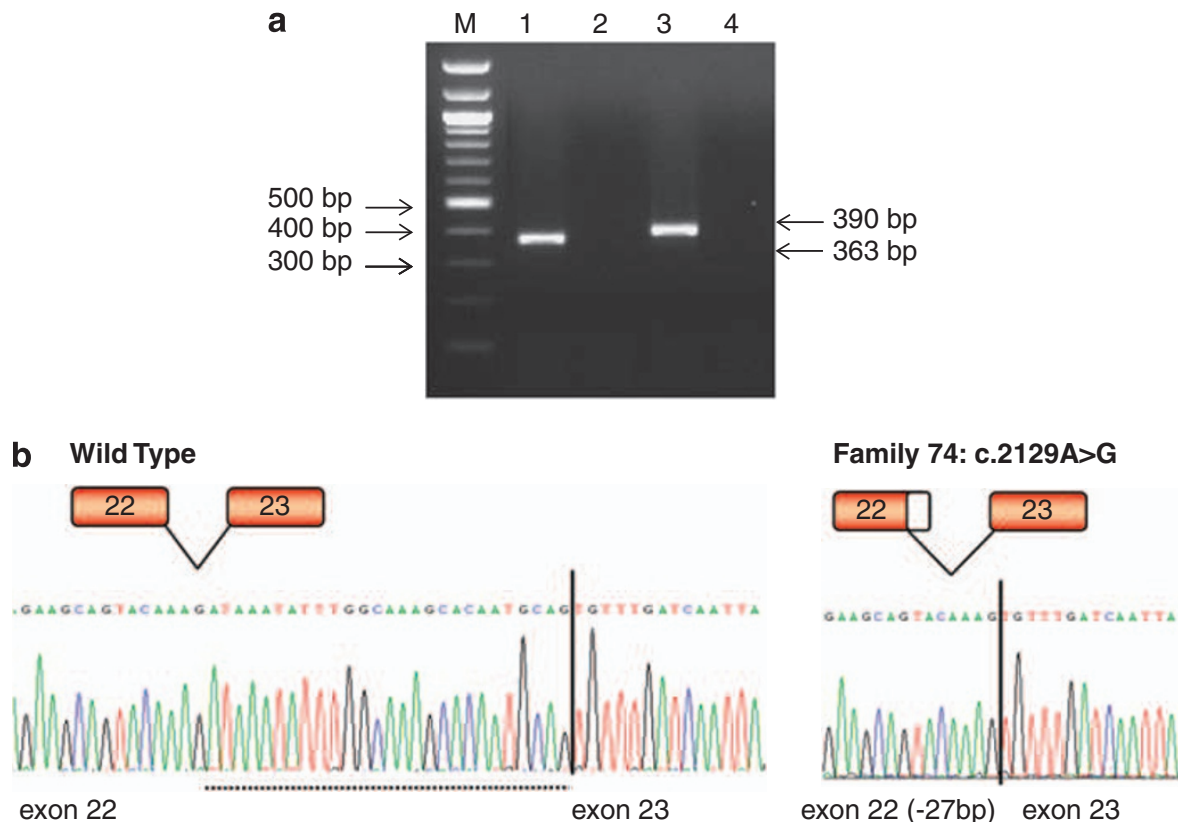

Figure 1 Reverse transcriptase PCR (RT-PCR) analysis on Family 74. (a) RT-PCR analysis of cDNA from a lymphoblastoid cell line from the proband of family 74 and wild-type control. Forward primer was designed from exon 21, 5'-CTGGCATTCGATTCAGAGTTGGTG-3', and reverse primer from exon 24, 5'-CGCAAACCGGTCTGGGTGCTTTG-3'. Lanes 1 and 3: affected male and control male, respectively; Lanes 2 and 4: no RT controls. (b) Sequence traces from the lymphoblastoid cell line showing the $27 \mathrm{bp}$ deletion compared with control lymphoblastoid cell line cDNA. The dotted line indicates the deleted bases in the abnormal transcript. 


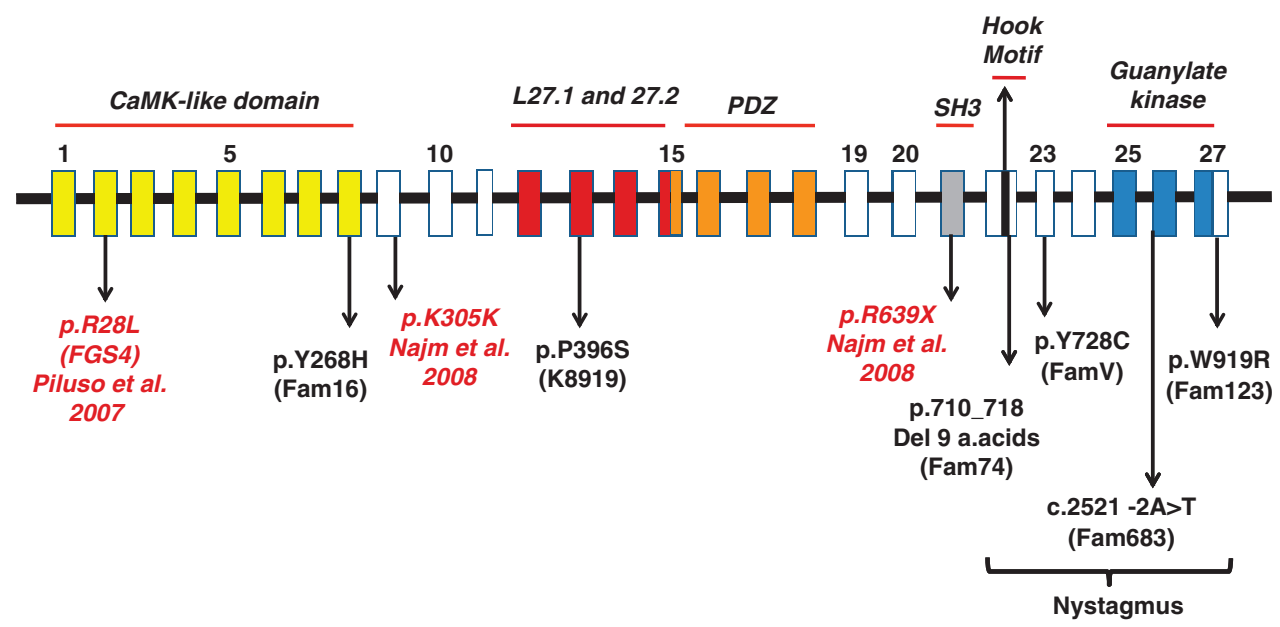

Figure 2 Schematic representation of the full-length CASK gene showing the different domains and both previously reported (in red) and new mutations (in black). The mutation nomenclature is based on the CASK full-length isoform encoding 926 amino acids (ENST00000378163). The four mutations in parentheses at the $\mathrm{C}$-terminal end are associated with nystagmus.

three affected individuals of the family available for study (Figure 3). Bioinformatic analysis did not indicate this variant as being pathogenic, yet the proline at position 396 is highly conserved (Figure 4).

Cohort of individuals with MR \pm nystagmus and microcephaly A missense variant and a splice site variant were identified in two of the 45 patients undergoing DNA sequence analysis of the coding exons of the CASK gene.

Family V. An A to G change at position 2183 (c.2183A $>$ G) in exon 23 was identified, resulting in the substitution of a cysteine for a tyrosine at position 728 (p.Y728C). The variant segregated in the family with MR and nystagmus (Figure 3). Polyphen and SIFT analysis of this variant predicts it to be probably damaging and not tolerated (Supplementary Table 1). The tyrosine at position 728 is highly conserved (Figure 4).

Family 683. An A to T change at the $3^{\prime}$ acceptor splice site sequence of exon 26, c.2521-2A $>$ T, was identified. RT-PCR analysis on the RNA isolated from lymphocytes from the proband showed that two transcript species were detected (Figure 5): (a) exon 25 spliced to exon 27 (exon 26 skipped), which results in an in-frame deletion of 28 amino acids; and (b) exon 25 spliced to exon 26 using an alternate $3^{\prime}$ acceptor sequence, which results in an in-frame deletion of three amino acids. RT-PCR performed on total RNA from lymphocytes from the obligate carrier, II-2, has the wild-type and both mutant transcripts (data not shown). Splice site calculations for the $3^{\prime}$ acceptor site and the alternate 3 'acceptor sequence revealed that the variants resulted in a decrease in information content from 7.77 to -0.60 and an increase from -6.06 to $0.82 .{ }^{17}$ This variant is present in the guanylate kinase domain (Figure 2).

Control data. The complete coding region of CASK was sequenced in 390 control X chromosomes. No missense variants were identified. ${ }^{6}$ No alterations were identified in a further 717 and 295 normal X chromosomes screened for the c.2183A $>\mathrm{G}$ variant and the c.2521$2 \mathrm{~A}>\mathrm{T}$ splice site variant, respectively. For the c. $1186 \mathrm{C}>\mathrm{T}$ variant, an additional $1287 \mathrm{X}$ chromosomes from de-identified normal individuals were screened, including 860 African Americans. The variant was present in one African-American female. The XCI status of this individual was uninformative and further clinical follow-up was not possible.

XCI studies. The XCI studies were performed on 11 carrier females (Table 1). Three females were uninformative. Seven, including III-6 from family K8919, with severe MR showed random XCI. Individual II-3 from family V, with mild MR and nystagmus, showed skewed XCI with a ratio of 90:10.

\section{Clinical reports}

Unless otherwise stated, all affected individuals were the product of normal pregnancies, were born at term and had normal birth weights. A summary of the clinical data is provided in Table 1 . Family pedigrees are shown in Figure 3.

Family 74. This family consists of four males with MR. The proband, IV-1, was noted to have nystagmus and unilateral strabismus as an infant. He attended a special school and, as an adult, functioned in the mild MR range. At 42 years of age, he was non-dysmorphic, with normal growth parameters. He had a resting hand tremor, accentuated with sustained posture. Detailed eye assessment including ocular motility testing and anterior segment examination and electrophysiology (pattern-onset visual-evoked potential and fullfield electroretinogram (ERG)) showed horizontal nystagmus in all positions of gaze. Visual acuity (VA) was $6 / 24$ in the right eye and 6/18 in the left eye.

Individuals III-3 and III-12 were cousins who shared many similar characteristics. Both had mild MR, were obese men of average height with a head circumference of $58 \mathrm{~cm}$ (98th centile). Both men had near-normal vision and a slow-beat gaze-evoked horizontal nystagmus, which was present in all directions of gaze. III-3 had a divergent strabismus, a resting tremor with an intention component and was mildly unsteady on his feet. The brain MRI of III-12 was normal.

Individual III-6 was said to have learning problems as a child and, as an adult, functioned at the level of mild MR. An examination at the 
Family 74 (p.D710G)

I

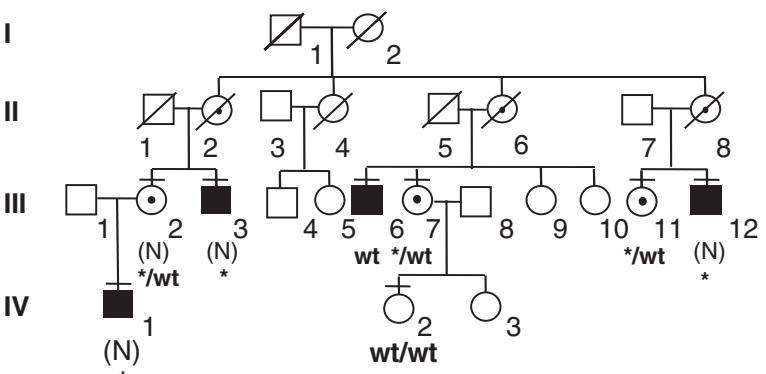

Family 16 (p.Y268H)

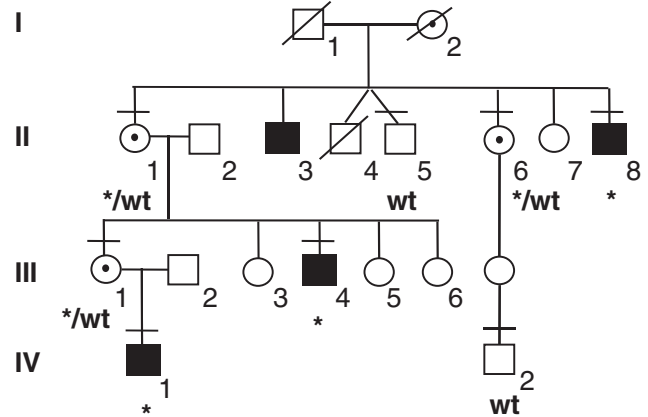

Family V (p.Y728C)

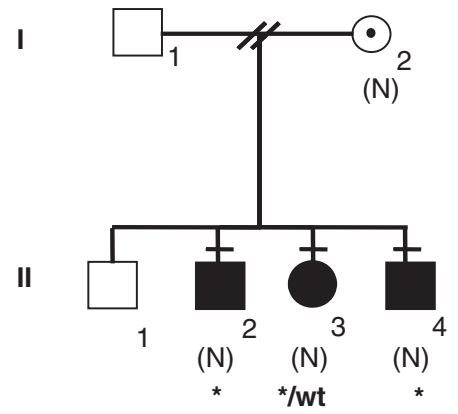

Family 123 (p.W919R)

I

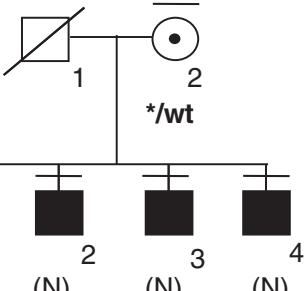

wt/wt $\quad(\mathrm{N}) \quad(\mathrm{N}) \quad(\mathrm{N})$

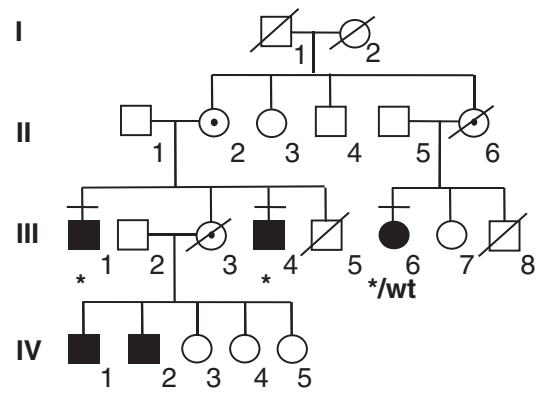

Family 683 (c.2521-2A $>T$ )

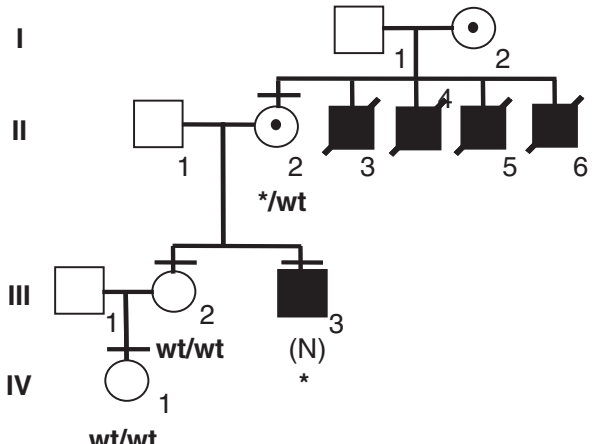

wt/wt

Figure 3 Family pedigrees of the six families with mutations in the CASK gene. Males are indicated by boxes and females by circles. Individuals with intellectual disability are shown as black boxes and circles. Carriers (either obligate or by testing) are shown with a dot within the circle. The letter ' $\mathrm{N}$ ' in parentheses indicates individuals with nystagmus. The horizontal bar above the symbols indicates individuals who underwent DNA analysis. Mutant $(*)$ and wild-type (wt) alleles are indicated below the symbols.

\begin{tabular}{|c|c|c|c|c|}
\hline & $\begin{array}{c}\mathrm{p} . \mathrm{Y} 268 \mathrm{H} \\
\downarrow\end{array}$ & $\begin{array}{c}\text { P.P396s } \\
\downarrow\end{array}$ & P.D710G & p.w919R \\
\hline Homo sapiens & PAERITVYEALNHPWLKERD. & . LYDKIN-TKSSPQIRNPPSDAVQR. & . .WFGKKKKQYKDKYLAKHNAVFDQLDLVTYEEVVKLP - - - -AFKR. & ....AVELVCTAPQWVPVSWVY \\
\hline Pan troglodytes & PAERITVYEALNHPWLKERD. & . LYDKIN-TKSSPQIRNPPSDAVQR . & . WFGKKKKQYKDKYLAKHNAVFDQLDLVTYEEVVKLP- - - - AFKR. & ... AVELVCTAPQWVPVSWVY \\
\hline Macaca mulatta & PAERITVYEALNHPWLKERD. & . LYDKIN-TKSSPQIRNPPSDAVQR. & . WFGKKKKQYYKDKYLAKHNA - - - -DLVTYEEVVKLP- - - AFKR. & ... AVELVCTAPQWVPVSWVY \\
\hline Mus musculus & PAERITVYEALNHPWLKERD. & . . LYDKIN-TKSSPQIRNPPSDAVQR. & . .WFGKKKKQYKDKYLAKHNAVFDQLDLVTYEEVVKLP- - - -AFKR. & ... AIELVCTASQWVPVSWVY \\
\hline Rattus norvegicus & PAERITVYEALNHPWLKERD. & . LYDKIN-TKSSPQIRNPPSDAVQR. & . .WFGKKKKQYKDKYLAKHNAVFDQLDLVTYEEVVKLP- - - -AFKR. & ... AVELVCTAPQWVPVSWVY \\
\hline Canis familiaris & PAERITVYEALNHPWLKERD. & . LYDKIN-TKSSPQIRNPPSDAVQR. & . . WFGKKKKQYYKDKYLAKHNAVFDQLDLLVTYEEVVKLP- - - -AFKR. & ... AVELVCTAPQWVPVSWVY \\
\hline Bos taurus & PAERITVYEALNHPWLKERD. & . LYDKIN-TKSSPQIRNPPSDAVQR . & . . WFGKKKKQYKDKYLAKHNAVFDQLDLVTYEEVVKLP - - - -AFKR. & .... AVEHVCTAPQWVPVSWVY \\
\hline Gallus gallus & PAERITVYEALNHPWLKERD. & . LYDKIN-TKSSPQIRNPPSDAVQR. & . WFGKKKKQYKDKYLAKHNA - - - - DLVTYEEVVKLP - - - -AFKR. & ... AVELVCTAPQWVPVSWVY \\
\hline X.tropicalis & PAERITVYEALNHPWLKERD. & . LYDKIN-TKSSPQIRNPPSDAVQR. & . . WFGKKKKQYKDKYLAKHNAVFDQLDLVTYEEVVKLP- - - -AFKR. & ... AIELVCTAPQWVPVSWVY \\
\hline Danio rerio & PAERITVYEALNHPWLK-RD. & . LYDKIN-TKSSPQIRNPSSDAVQR. & . . WFGKKKKQYKDKYLAKHNAVFDQLDLVTYEEVVKLP - - - -AFKR. & ... AIDMVCSTAQWVPVSWVY \\
\hline D. melanogaster & PHHRLSITEVLDHPWIRDRD. & . LFDRIAATVVTSNGRAPAAEAVGR & . . IFGRKKKQCRDKYLAKHNAIFDTLDVVTYEEVVKVPVGDPNFQR. & ... AIDRVHTTPQWVPVSWLY \\
\hline C. elegans & PSKRISAKEALNHEWIRDKE & . LYDKIKA-LPCEPVVTE-VDTSTL. & . . WFNKKKKYYTTKYLQKHSALFDQLDLVTYEEVMRLS - - - - QYRR. & ... LVEKLPAYPQWLPVTWVY \\
\hline
\end{tabular}

Figure 4 Protein alignment of CASK orthologues. Homo sapiens (ENSP00000367405), Pan troglotydes (ENSPTRP00000047442), Macaca mulatta (ENSMMUP00000041117), Mus musculus (ENSMUSP00000112322), Rattus norvegicus (ENSRNOP00000004187), Canis familiaris (ENSCAFP00000021243), Bos taurus (ENSBTAP00000026825), Gallus gallus (ENGALP000000026115), Xenopus tropicalis (ENSXET00000010527), Danio rerio (ENSDARP00000091622), Drosophila melanogaster (FBPP0083559) and Caenorhabditis elegans (F17E.5.1a). Missense mutations are indicated by arrows and red letters. Amino acids that are not conserved are shown in blue. The protein 4.1 binding motif (Hook motif) is underlined in the Homo sapiens sequence. The deleted amino acids (p.710_718) in family 74 are indicated by bracket. 


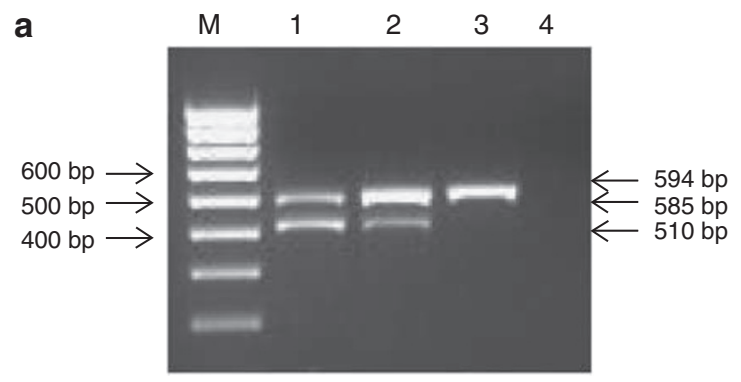

b
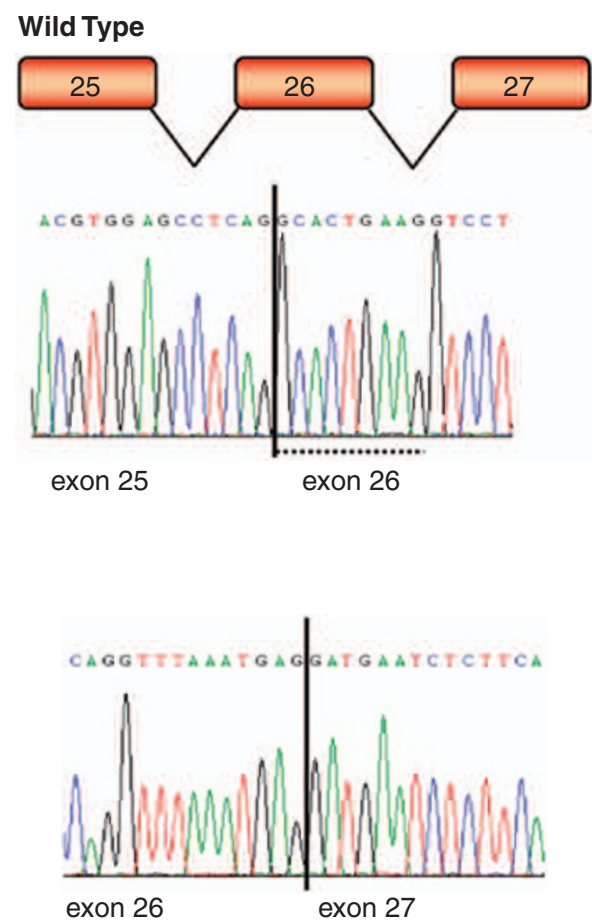

Family 683: c.2521-2A $>T$
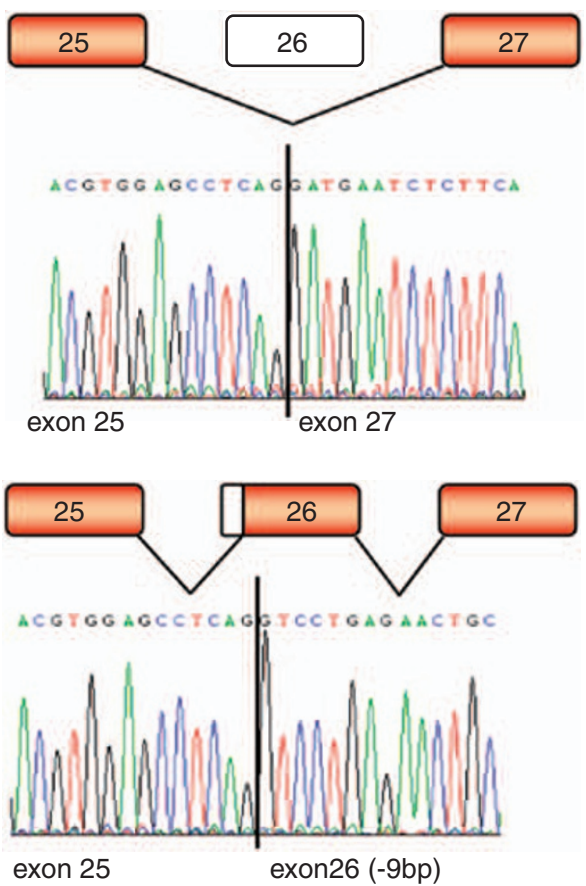

Figure 5 Reverse transcriptase PCR analysis of Family 683. (a) Lane 1: affected male; Lane 2: obligate carrier; Lane 3: wild-type control; and Lane 4: negative control. Forward primer was designed from exon 24, 5'-TCATCACAAAGCACCCAGAC-3', and reverse primer was from the 3'-UTR, 5'CCATGACCTGCTGACACAAG-3'. (b) Sequence traces showing the wild-type junction sequence between exons 25-26 and exons 26-27 and the two abnormal transcripts observed in family 683 as a result of splice mutation (c.2521-2A $>T$ ). The dotted line indicates the deleted bases in the abnormal transcript.

age of 59 years showed a non-dysmorphic obese man of average height and head circumference.

Three carriers, III-2, III-7 and III-11, were all of normal intelligence. Clinical examinations of III-7 and III-11 were unremarkable. Aged 67 years, III-2 had a mild resting hand tremor, accentuated with sustained posture. Ocular assessment identified bilateral mature cataracts, horizontal nystagmus in all positions of gaze and a gaze-evoked component. Her ERG showed reduced scotopic responses, consistent with her cataracts. ${ }^{18}$ VA was $6 / 24$ in the right eye and hand movements in the left eye.

Family 16. The family consists of four affected males. The proband, IV-1, toe walked because of tight achilles tendons. He had severe-toprofound MR and a seizure disorder. His behaviour was within the autistic spectrum. He had normal growth parameters and was nondysmorphic. There was no evidence of nystagmus in him or in his extended family. The three carrier females, II-1, II-6 and III-1, were of normal intelligence.
His uncle, III-4, has severe MR and a seizure disorder. He was placed in an institution at the age of 5 years and was described by the family as being similar to the proband. The proband's great uncle, II-3, was also a toe walker, had obsessive behaviours, developed only a few words and is in full-time residential care.

Family 123. This family consists of three affected brothers, who all attended special schools and, as adults, function in the mild MR range. Nystagmus was noted in childhood. At the age of 17 years, II-2 developed a generalised seizure disorder. All three were tall and nondysmorphic with normal head circumferences. All had stable nearnormal vision, and a slow-beat gaze-evoked horizontal nystagmus, which was present in all directions of gaze. Their mother, I-2, had a fine tremor which increased with sustained posture. Her physical examination was otherwise normal.

K8919 (family 245). This African-American family consists of four males and one female with MR. III-1 had profound MR, with an 
Table 1 Clinical data summary of patients with CASK mutations

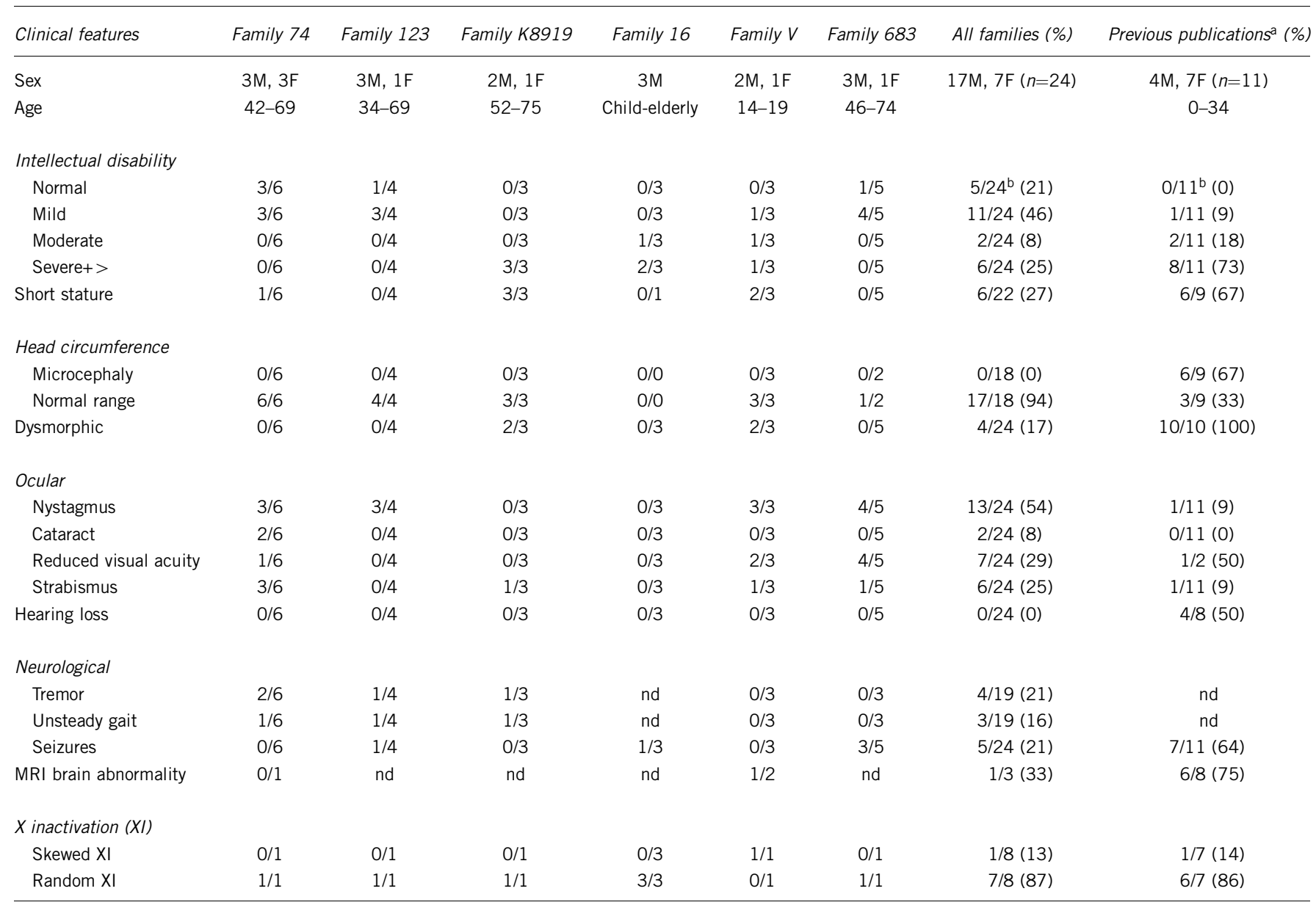

nd, not done.

a Hayashi et al, 200833 Najm et al, 20084; Froyen et al, 2007²; Piluso et al, 2003, $2009^{5}$

benominator specified according to the number of patients for whom information was available.
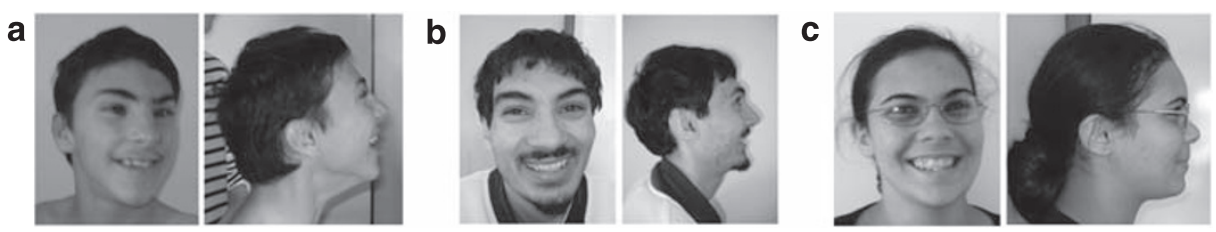

Figure 6 Faces of affected individuals with dysmorphic facial features. (a-c) Family V: The proband, II-4 (a), and his brother, II-2 (b), both show microcephaly, high nasal bridge, upslanting palpebral fissures and a short columella. Their sister, II-3 (c), is non-dysmorphic.

IQ of 7. At 58 years of age, his height was $152 \mathrm{~cm}(<3 \mathrm{rd}$ centile) and head circumference was $56 \mathrm{~cm}$ (50th centile). He had a flat mid face, open mouth and eversion of the lower lip. Neurological examination showed an unsteady gait and a resting tremor.

His brother, III-4, had severe MR. Aged 52 years, his height was $147 \mathrm{~cm}(<3 \mathrm{rd}$ centile) and head circumference was $56 \mathrm{~cm}$ (50th centile). Dysmorphic facial features included a flat nasal bridge, anteverted nares, wide mouth, broad shallow palate, broad and grooved tongue and a short and wide neck. Convergent strabismus was present.

III-6 had severe MR, with an IQ of 33. She was non-dysmorphic with a height of $151.1 \mathrm{~cm}$ (3rd centile) and head circumference of $54 \mathrm{~cm}$ (35th centile).
Family $V$. This family consists of two affected males and their sister. The proband, II-4, had severe MR, which was formally assessed at the age of 3 years. A brain MRI at the age of 5 years showed cerebellar hypoplasia and pachygyria. Ophthalmologist reviews demonstrated congenital nystagmus, a slow-beat gaze-evoked horizontal nystagmus present in all directions of gaze, significant convergent strabismus, reasonable VA and bilateral mild temporal disc pallor. Aged 14 years, he was thin, with a height of $140 \mathrm{~cm}(<3 \mathrm{rd}$ centile) and head circumference of $48.5 \mathrm{~cm}$ (3rd centile). Dysmorphic facial features included synophyrys, a high nasal bridge, upslanting palpebral fissures and a short columella (Figure 6a).

His older brother, II-2, had moderate MR, with an IQ of 38-50. Ophthalmologist review diagnosed congenital motor nystagmus 
associated with stable mild reduced vision, mild astigmatism, no fundal abnormalities and a slow-beat horizontal nystagmus present in all directions of gaze and accentuated by lateral gaze. Aged 19 years, he was thin, with a height of $169 \mathrm{~cm}(<3 \mathrm{rd}$ centile) and head circumference of $53.2 \mathrm{~cm}(2-10$ th centile). Dysmorphic facial features were similar to that of his brother (Figure $6 \mathrm{~b}$ ).

Their sister (II-3) had mild MR, formally assessed at the age of 14 years. Ophthalmologist reviews demonstrated congenital nystagmus and astigmatism with a VA of 6/24 in each eye. Detailed eye assessment at the age of 19 years demonstrated a slow-beat horizontal nystagmus present in all directions of gaze, normal scotopic ERG and no retinopathy. A brain MRI was normal. Growth parameters were normal and there was no dysmorphism (Figure 6c). Their mother, I-2, reportedly had some cognitive difficulties. Nystagmus had been noted for many years.

Family 683. This family consists of four affected males with mild MR. The proband, III-3, was noted to have nystagmus at 3 weeks. He attended a special school and had infrequent absence seizures. Aged 46 years, he lives with his mother and is able to self-care. He is partially sighted with significant horizontal nystagmus and high myopia. He is of an average build with height of $166 \mathrm{~cm}$ (10th centile) and head circumference of $60 \mathrm{~cm}$ (>98th centile). He is non-dysmorphic and had a normal neurological examination. Both II-2 and I-2 had normal intelligence and normal ocular examinations.

Individual II-2 had three affected brothers, II-3, II-4 and II-5, all with mild MR, although only II-4 managed supervised employment. They lived together with their mother and were able to care for themselves. II-3 and II-5 developed epilepsy in childhood. Ophthalmic assessment showed all three had congenital pendular nystagmus, mildly reduced vision and wore corrective glasses. II-3 had an alternating divergent strabismus, right myopic astigmatism and left mixed astigmatism. II-4 had high myopia, pallor of the optic discs, minimal pigmentary stippling of macula and peripheral retinas, normal ERG, normal electro-oculogram and diminished amplitude of the visual-evoked potential.

\section{DISCUSSION}

In this study, we characterised six mutations in the CASK gene, associated with diverse clinical phenotypes. Two of these, p.D710G (family 74) and c.2521-2A $>\mathrm{T}$ (family 683), resulted in errors of mRNA processing. The p.D710G mutation results in alternative splicing of exon 22, causing an in-frame deletion of nine amino acids at the C-terminal end of the 'Hook Motif' (the protein 4.1 binding motif). The c.2521-2A $>\mathrm{T}$ mutation within the $3^{\prime}$ acceptor splice site sequence of exon 26 results in two alternative splice site transcripts. Both result in in-frame deletions (p.841_868 and p.841_843 del ALK) within the C-terminal guanylate kinase domain, a pseudokinase that, in neurons, is involved in re-targeting the CASK protein to the nucleus, where it interacts with the transcription factor TBR-1 (T-brain-1) and nucleosome-assembly protein CASK-interacting nucleosome assembly protein (CINAP). These proteins form a transcriptional complex that regulates expression of genes, such as RELN and NR2b (NMDA receptor $2 \mathrm{~b}$ ), which are important for neuronal function and development. ${ }^{7,9,19,20}$ Huang and Hsueh ${ }^{20}$ recently demonstrated that a point mutation in the Thr724 residue of the rat CASK guanylate kinase domain reduced the interaction between CASK and both Tbr-1 and CINAP proteins. We postulate that the above splice mutations would result in a similar or more pronounced effect.
In contrast to the previously reported highly deleterious variants associated with inviability or neonatal lethality in males, mutations compatible with male viability are likely to have a relatively mild impact on protein structure and function. Thus, one might expect that missense variants may not be clearly flagged as deleterious with Panther/Polyphen-type prediction programmes and that the information obtained should be interpreted in light of all available information. Bioinformatic analyses (Supplementary Table 1) of the four missense mutations showed that all were predicted to be destabilising using iPTREE analysis. The p.W919R mutation (family 123) results in the substitution of arginine, a charged amino acid, for tryptophan, a polar amino acid. SIFT and Polyphen analysis predicted that p.W919R and p.Y728C (family V) mutations would not be tolerated and probably damaging. Both amino acids are highly conserved in different species (Figure 4). Panther prediction was not deleterious for the other two mutations, p.Y268H (family 16) and p.P396S (family K8919). Yet, the p.P396S missense mutation involves a highly conserved site within the L27.1 domain, which specifically binds to the unique L27 domain of SAP97, in turn having an important role in the establishment and maintenance of neuronal and epithelial cell polarisation. ${ }^{9}$ The p.Y268H mutation occurs at a highly conserved amino acid position within the CaMK-like domain. The CaMK-like domain binds to several proteins, such as Caskin $1^{21}$ and Mint1, ${ }^{22,23}$ and adopts a constitutively active conformation that binds to ATP and catalyses the transfer of a phosphate group without $\mathrm{Mg} 2^{+} .{ }^{24}$ The lack of sequence variants of the missense mutations in control samples also suggests that the evolutionary constraints on CASK are stringent and that these mutations are unlikely to be polymorphisms, but this cannot be completely excluded.

The most obvious explanation for the striking difference in clinical severity between individuals with CASK mutations is that hemizygous missense variants are likely to be less deleterious than inactivating mutations. Our families exhibited widely variable phenotypes, including mild non-syndromic XLMR and XLMR with nystagmus \pm dysmorphic facial features. In stark contrast to the severely affected individuals reported by Najm et al, ${ }^{4}$ our carrier females were either phenotypically normal or less severely affected compared with the males within their family; three of our families had only mild MR, no affected individual had microcephaly and only one of the three individuals undergoing brain MRI exhibited brain malformations or cerebellar hypoplasia. Similar to the recently reported family, ${ }^{5}$ macrocephaly was present in one affected male and relative macrocephaly was seen in two of the affected males from family 74 . The four families with nystagmus (families 74, 123, V and 683), all have mutations within the C-terminal of the CASK protein, suggesting a possible genotype-phenotype correlation for the presence of nystagmus (Figure 3).

Nystagmus is an unusual and distinctive clinical feature, not commonly seen in association with MR. Exceptions to this include Pelizaeus-Merzbacher disease ${ }^{25,26}$ and chromosome 18q22.3-q23 deletions, ${ }^{27}$ both of which may be associated with nystagmus. Nystagmus can be associated with various diseases, such as vestibular or cerebellar dysfunction or may be inherited, either as an isolated finding (congenital idiopathic/motor nystagmus) or related to sensory defects of the retina or anterior visual pathways, such as albinism or retinal dystrophy. ${ }^{28}$ A single gene, FRMD7 at Xq26.2 (NYS1), has been identified as causing congenital nystagmus, ${ }^{29-31}$ although other loci, including NYS5, NYS2 at 6p12, NYS3 at 7p11, NYS4 at 13q31-q33 and $18 \mathrm{q}$ exist. ${ }^{27,32-36}$

Detailed eye assessments of individuals from families 74, V and 683 demonstrated many common features, such as early age of onset of 
nystagmus, horizontal nystagmus present in all directions of gaze, pendular or increasing velocity waveforms, mildly reduced VA and absence of sensory defects. The consistent finding of gaze-evoked nystagmus indicates a dysfunction of the neural integrator, which is required for all conjugate eye movements. The neural integrator is a term applied to an ocular motor function carried out by groups of cells in the cerebellum, the prepositus hypoglossus and medial vestibular nucleus. ${ }^{37-39}$ Thus, the anatomical localisation of neural integrator dysfunction provides a plausible explanation for the ocular signs (nystagmus \pm other ocular deficits, including retinal anomalies and strabismus) occurring in patients with CASK mutations. ${ }^{2-4}$ Nystagmus does not seem to be related to cerebellar dysfunction, as the majority of patients reported by Najm et al had cerebellar hypoplasia, yet none had nystagmus, and of our three individuals with nystagmus who had MRI brain scans, only one (II-2, family V) had mild cerebellar hypoplasia. Moreover, there was no obvious clinical cerebellar dysfunction in the majority of individuals with nystagmus. Further studies are needed to determine whether mutations within the $\mathrm{C}$-terminal end of the CASK gene affect development or function of the neural integrator.

Despite over 90 XLMR genes having been identified, each gene accounts for only a small proportion of XLMR cases, and the causative mutation in many XLMR families remains unknown. ${ }^{40}$ Of the four families in whom CASK mutations were identified from 358 XLMR probands screened for mutations, ${ }^{6}$ two had the unusual and distinctive clinical feature of nystagmus. An additional two mutations were identified in the cohort of 45 patients with MR and nystagmus. Although the numbers are small, it suggests that consideration should be given to screening for CASK mutations in individuals with MR and nystagmus. Together, 6 out of the 403 (1.5\%) individuals screened had a mutation within the CASK gene.

In summary, we characterised six novel sequence alterations within the CASK gene and postulate that a milder phenotypic spectrum is associated with missense mutations, compared with inactivating mutations. A genotype-phenotype correlation seems to be likely for the presence of nystagmus, with mutations situated at the C-terminal of the CASK protein. CASK mutations are likely to represent a relatively frequent cause of XLMR, with a diverse clinical spectrum, ranging from non-syndromic XLMR to severe MR associated with microcephaly, brain malformations and facial dysmorphism.

\section{CONFLICT OF INTEREST}

The authors declare no conflict of interest.

\section{ACKNOWLEDGEMENTS}

We express our gratitude to the families for their participation in this study. We thank Cindy Skinner for sample coordination and acknowledge the technical assistance of Melissa Cook for sequencing. This work was supported in part by the Australian National Health and Medical Research Council program grant 400121, the New South Wales (NSW) Health Department, through the support of the NSW GOLD service, the Wellcome Trust; MRC; Action Medical Research, NIHR; National Institute of Child health and Human Development grant (HD260202) to CES and a grant from South Carolina Department of Disabilities and Special Needs (SSDDSN). This paper is dedicated to the memory of Ethan Francis Schwartz 1996-1998.

\section{Web Resources}

http://www.nei.nih.gov/news/statements/cemas.pdf

PolyPhen, http://genetics.bwh.harvard.edu/pph/

SIFT, http://blocks.fhcrc.org/sift/SIFT.html

PANTHER, http://www.pantherdb.org/

iPTREE-STAB, http://210.60.98.19/IPTREEr/iptree.htm
1 Dimitratos SD, Stathakis DG, Nelson CA, Woods DF, Bryant PJ: The location of human CASK at Xp11.4 identifies this gene as a candidate for X-linked optic atrophy. Genomics 1998; 51: 308-309.

2 Froyen G, Van Esch H, Bauters M et al: Detection of genomic copy number changes in patients with idiopathic mental retardation by high-resolution $\mathrm{X}$-array-CGH: important role for increased gene dosage of XLMR genes. Hum Mutat 2007; 28: 1034-1042.

3 Hayashi S, Mizuno S, Migita $\mathrm{O}$ et al: The CASK gene harbored in a deletion detected by array-CGH as a potential candidate for a gene causative of X-linked dominant mental retardation. Am J Med Genet A 2008; 146A: 2145-2151.

4 Najm J, Horn D, Wimplinger I et al: Mutations of CASK cause an X-linked brain malformation phenotype with microcephaly and hypoplasia of the brainstem and cerebellum. Nat Genet 2008; 40: 1065-1067.

5 Piluso G, D'Amico F, Saccone $V$ et al: A missense mutation in CASK causes FG syndrome in an Italian family. Am J Hum Genet 2009; 84: 1-16.

6 Tarpey PS, Smith R, Pleasance E et al: A systematic, large-scale resequencing screen of the $X$ chromosome coding exons in mental retardation. Nat Genet 2009; 41: 535-543.

7 Hsueh YP, Wang TF, Yang FC, Sheng M: Nuclear translocation and transcription regulation by the membrane-associated guanylate kinase CASK/LIN-2. Nature 2000; 404: 298-302.

8 Stevenson D, Laverty HG, Wenwieser S, Douglas M, Wilson JB: Mapping and expression analysis of the human CASK gene. Mamm Genome 2000; 11: 934-937.

9 Hsueh YP: The role of the MAGUK protein CASK in neural development and synaptic function. Curr Med Chem 2006; 13: 1915-1927.

10 Hevner R, Shi L, Justice $N$ et al: Tbr 1 regulates differentiation of the preplate and layer 6. Neuron 2001; 29: 353-366.

11 Bulfone A, Wang F, Hevner R et al: An olfactory sensory map develops in the absence of normal projection neurons or GABAergic interneurons. Neuron 1998; 21: 1273-1282.

12 Leid M, Ishmael J, Avram D, Shepherd D, Fraulob V, Dolle P: CTIP1 and CTIP2 are differentially expressed during mouse embryogenesis. Gene Expr Patterns 2004; 4: 733-739.

13 Crawford J, Lower KM, Hennekam RCM et al: Mutation screening in BörjesonForssman-Lehmann syndrome: identification of a novel de novo PHF6 mutation in a female patient. J Med Genet 2006; 43: 238-243.

14 Wimplinger I, Morleo M, Rosenberger $\mathrm{G}$ et al: Mutations of the mitochondrial holocytochrome c-type synthase in X-linked dominant microphthalmia with linear skin defects syndrome. Am J Hum Genet 2006; 79: 878-889.

15 Tarpey P, Parnau J, Blow M et al: Mutations in the DLG3 gene cause nonsyndromic Xlinked mental retardation. Am J Hum Genet 2004; 75: 318-324.

16 Raymond F, Tarpey P, Edkins S et al: Mutations in ZDHHC9, which encodes a palmitoyltransferase of NRAS and HRAS, cause X-linked mental retardation associated with a Marfanoid habitus. Am J Hum Genet 2007; 80: 982-987.

17 Yeo G, Burge C: Maximum entropy modeling of short sequence motifs with applications to RNA splicing signals. J Comput Biol 2004; 11: 377-394.

18 Cruz RD, Adachi-Usami E: Quantitative evaluation of electroretinogram before cataract surgery. Jpn J Ophthalmol 1989; 33: 451-457.

19 Wang GS, Hong CJ, Yen TY et al: Transcriptional modification by a CASK-interacting nucleosome assembly protein. Neuron 2004; 42: 113-128.

20 Huang T-N, Hsueh Y-P: CASK point mutation regulates protein-protein interactions and NR2b promoter activity. Biochem Biophys Res Commun 2009; 382: 219-222.

21 Tabuchi K, Biederer T, Butz S, Sudhof TC: CASK participates in alternative tripartite complexes in which Mint 1 competes for binding with Caskin 1, a novel CASK-binding protein. J Neurosci 2002; 22: 4264-4273.

22 Butz S, Okamoto M, Sudhof TC: A tripartite protein complex with the potential to couple synaptic vesicle exocytosis to cell adhesion in brain. Cell 1998; 94: 773-782.

23 Borg JP, Straight SW, Kaech SM et al: Identification of an evolutionarily conserved heterotrimeric protein complex involved in protein targeting. J Biol Chem 1998; 273 : 31633-31636.

24 Mukherjee K, Sharma M, Urlaub $\mathrm{H}$ et al: CASK Functions as a Mg2+-independent neurexin kinase. Cell 2008; 133: 328-339.

25 Inoue K: PLP1-related inherited dysmyelinating disorders: Pelizaeus-Merzbacher disease and spastic paraplegia type 2. Neurogenetics 2005; 6: 1-16.

26 Sistermans E, de Coo R, De Wijs I, Van Oost B: Duplication of the proteolipid protein gene is the major cause of Pelizaeus-Merzbacher disease. Neurology 1998; 50: 1749-1754.

27 Linnankivi T, Tienari $\mathrm{P}$, Somer $\mathrm{M}$ et al: $18 \mathrm{q}$ deletions: clinical, molecular, and brain MRI findings of 14 individuals. Am J Med Genet A 2006; 140: 331-339.

28 Leigh RJ, Zee DS: The Neurology of Eye Movements, 4th edn. New York: Oxford University Press, 2006

29 Tarpey P, Thomas S, Sarvananthan N et al: Mutations in FRMD7, a newly identified member of the FERM family, cause X-linked idiopathic congenital nystagmus. Nat Genet 2006; 38: 1242-1244.

30 Self JE, Shawkat F, Malpas CT et al: Allelic variation of the FRMD7 gene in congenital idiopathic nystagmus. Arch Ophthalmol 2007; 125: 1255-1263.

31 Zhang B, Liu Z, Zhao G et al: Novel mutations of the FRMD7 gene in X-linked congenital motor nystagmus. Mol Vis 2007; 13: 1674-1679.

32 Kerrison J, Koenekoop R, Arnould V, Zee D, Maumenee I: Clinical features of autosomal dominant congenital nystagmus linked to chromosome 6p12. Am J Ophthalmol 1998; 125: $64-70$. 
33 Klein C, Vieregge P, Heide W et al: Exclusion of chromosome regions $6 \mathrm{p} 12$ and 15q11, but not chromosome region $7 \mathrm{p} 11$, in a German family with autosomal dominant congenital nystagmus. Genomics 1998; 54: 176-177.

34 Ragge NK, Hartley C, Dearlove AM, Walker J, Russell-Eggitt I, Harris CM: Familial vestibulocerebellar disorder maps to chromosome 13q31-q33: a new nystagmus locus. J Med Genet 2003; 40: 37-41.

35 Hertle R, Yang D, Kelly K, Hill V, Atkin J, Seward A: X-linked infantile periodic alternating nystagmus. Ophthalmic Genet 2005; 26: 77-84.

36 Guo X, Li S, Jia X, Xiao X, Wang P, Zhang Q: Linkage analysis of two families with X-linked recessive congenital motor nystagmus. J Hum Genet 2006; 51: 76-80.
37 Cannon S, Robinson D, Shamma S: A proposed neural network for the integrator of the oculomotor system. Biol Cybern 1983; 49: 127-136.

38 Buttner-Ennever J, Horn A, Graf W, Ugolini G: Modern concepts of brainstem anatomy: from extraocular motoneurons to proprioceptive pathways. Ann N Y Acad Sci 2002; 956: 75-84.

39 Arnold D, Robinson D: A learning network model of the neural integrator of the oculomotor system. Biol Cybern 1991; 64: 447-454.

40 Gécz J, Shoubridge C, Corbett M: The genetic landscape of intellectual disability arising from chromosome X. Trends Genet 2009; 25: 308-316.

Supplementary Information accompanies the paper on European Journal of Human Genetics website (http://www.nature.com/ejhg)

\title{
Corrigendum to: CASK mutations are frequent in males and cause $\mathrm{X}$-linked nystagmus and variable XLMR phenotypes
}

\author{
Anna Hackett, Patrick S Tarpey, Andrea Licata, James Cox, Annabel Whibley, Jackie Boyle, Carolyn Rogers, \\ John Grigg, Michael Partington, Roger E Stevenson, John Tolmie, John RW Yates, Gillian Turner, \\ Meredith Wilson, Andrew P Futreal, Mark Corbett, Marie Shaw, Jozef Gecz, F Lucy Raymond, \\ Michael R Stratton, Charles E Schwartz and Fatima E Abidi
}

European Journal of Human Genetics (2010) 18, 552; doi:10.1038/ejhg.2010.24

Correction to: European Journal of Human Genetics (2010) 18, 554-552; doi:10.1038/ejhg.2009.220; published online 23 December 2009

Since the publication of the above paper, the authors have identified an error. In the Results section, under the sub-heading 'Sequencing of the CASK gene', Family 123, the first sentence is incorrect; 2756
(c.2756T $>$ C) should be replaced with 2755 (c.2755T $>$ C). The correct sentence is:

A $\mathrm{T}$ to $\mathrm{C}$ change in exon 27 at position 2755 (c.2755T >C) was identified, resulting in the substitution of arginine for tryptophan at position 919 (p.W919R) at the C-terminal end of the CASK protein (Figure 2).

The authors would like to apologise for this mistake. 